\title{
The diagnostic accuracy of liquid exosomes for lung cancer detection: a meta-analysis
}

This article was published in the following Dove Medical Press journal:

OncoTargets and Therapy

\author{
Zhipeng Song \\ Saisai Wang \\ Yang Liu \\ Department of Epidemiology, Beijing \\ Chest Hospital, Capital Medical \\ University, Beijing, China
}

\begin{abstract}
Purpose: Several studies have suggested that liquid exosomes can be used as biomarkers for the diagnosis of lung cancer (LC). The purpose of this meta-analysis was to investigate the comprehensive diagnostic value of liquid exosomes for LC.

Materials and methods: Relevant studies were searched from multiple electronic databases. The quality of the studies was assessed by the Quality Assessment of Diagnostic Accuracy Studies-2 criteria in RevMan 5.3 software. Stata 14.0 software and Meta-disc 1.4 software were used to synthesize the diagnostic parameters. Publication bias was judged according to the Deeks' funnel plot asymmetry test.

Results: There were 13 eligible articles that comprised 1,338 LC patients and 1,075 paired controls for the meta-analysis. The pooled sensitivity (SEN), specificity (SPE), diagnostic likelihood ratio positive (DLR+), diagnostic likelihood ratio negative (DLR-), diagnostic OR (DOR), and area under the curve (AUC) of liquid exosomes in diagnosing LC were 0.82 (95\% CI: 0.76-0.87), 0.84 (95\% CI: 0.77-0.89), 5.27 (95\% CI: 3.58-7.75), 0.21 (95\% CI: 0.15-0.29), 25.14 (95\% CI: 14.25-44.33), and 0.90 (95\% CI: 0.87-0.92), respectively. Research based on serum, miRNA, the isolation kit method, one index in exosomes, patient sample size of 50 or greater, and control group size of 50 or greater obtained higher AUC values when the LC type was small cell lung cancer.

Conclusion: Liquid exosomes have shown potential as novel biomarkers that could facilitate LC diagnosis. Further prospective studies are still needed to confirm the diagnostic value of liquid exosomes.
\end{abstract}

Keywords: lung neoplasm, liquid, exosomes, diagnosis, meta-analysis

\section{Introduction}

Lung cancer (LC) is one of the main malignant tumors that threatens human health worldwide, and it is a leading cause of morbidity and mortality. ${ }^{1}$ Clinically, LC is usually diagnosed by tissue biopsy, which is the gold standard for diagnosis. ${ }^{2}$ However, tissue biopsy is invasive, and it cannot be used as a routine method for outpatient and physical examination screening. ${ }^{3}$ Recently, low-dose spiral computed tomography has been used as a tool to screen high-risk groups for LC; it has achieved certain successes, ${ }^{4}$ but its false-positive rate and requirements for technicians pose substantial limitations. ${ }^{5}$ At the same time, liquid biopsy technology emerged, offering an alternative to historical diagnostic tools. Exosomes are cell-derived vesicles with diameters of 30-100 nm. They can be detected from various body fluids, including plasma, serum, saliva, urine, pleural effusion, cerebrospinal fluid, and semen. ${ }^{6}$ By detecting and analyzing the contents of tumor-derived exosomes, relevant information of tumor cells can be obtained. This method is expected to become a novel and effective diagnostic approach. ${ }^{7-9}$ 
Today, research of exosomes is quite popular, and studies regarding their application in LC diagnosis have been conducted in China as well as in other countries. ${ }^{10}$ However, the small sample size of each study limits the value of this research. High-quality evidence-based medical research is lacking, as well. In order to evaluate the diagnostic value of liquid exosomes for LC diagnosis, we collected published literatures and conducted a meta-analysis with an aim to promote the development of novel biomarkers for $\mathrm{LC}$ at the liquid biopsy level.

\section{Materials and methods}

\section{Literature search}

We referred to the PRISMA criteria to guide the implementation of this meta-analysis. ${ }^{11}$ Literature searches were conducted in PubMed, EMBASE, Cochrane Library, Web of Science, China National Knowledge Infrastructure, Wanfang databases, and VIP Journal Integration on Platform through July 22, 2018, with language restricted to English or Chinese. The key terms applied for the electronic databases were: ("lung" or "pulmonary" or "pulmonic" or "pneumonic" or "pneumal") and ("cancer" or "carcinoma" or "carcinomata" or "tumor" or "tumour" or "neoplasm") and ("exosomes" or "exosome") and ("diagnosis" or "diagnose"). In order to avoid missing related articles, an additional manual search was performed using the reference lists of relevant studies. The literature search was performed independently by two investigators and, in case of discordance, the search was compared to that of an additional investigator.

\section{Selection of studies}

Studies that met the following criteria were included in this study: 1) included all types of LC patients; 2) included patients with benign lung disease, healthy volunteers, or a population with a relevant negative maker as a control group; 3) evaluated a liquid sample type; 4) included relevant data about the diagnostic accuracy of exosomes for LC; and 5) provided $2 \times 2$ contingency tables that could be directly extracted or calculated from the reviewed literatures.

The following studies were excluded: 1) duplicate literatures; 2) articles with insufficient data; 3 ) animal studies, reviews, meta-analysis, letters, or expert opinions; 4) articles not related to exosomes or LC; and 5) articles not related to diagnostic value.

\section{Data extraction}

We extracted true-positive, false-positive, false-negative, and true-negative values from the diagnostic studies. When the data were unavailable, we used pooled sensitivities (SENs) and pooled specificities (SPEs) to calculate these data and completed the $2 \times 2$ tables. The first author, year of publication, research country, sample type, experimental method used for exosome isolation, type of exosome content, content number, type of LC, type of control group, and sample size were also recorded from the included studies. We contacted the authors to verify specifications when there were inconsistencies.

\section{Quality assessment}

The quality of the included literature was evaluated by Quality Assessment of Diagnosis Accuracy Studies (QUADAS)-2 criteria in RevMan 5.3 software. ${ }^{12}$ The QUADAS-2 tool is composed of four key domains: patient selection, index test, reference standard, and flow and timing. It includes 14 questions: the answer of risk for bias was rated as "no", "yes", or "unclear", which corresponded to scores of $-1,1$, and 0 , respectively, and the applicability concerns were judged as "low concern", "high concern", or "unclear concern". We eliminated the included studies with low quality. The quality of the literature was assessed by two investigators independently, and, in case of discordance, the conclusions were compared to that of an additional investigator.

\section{Statistical analysis}

Statistical analysis was conducted by Stata (version 14.0; Stata Corporation, College Station, TX, USA) and Meta-disc (version 1.4; XI Cochrane Colloquium, Barcelona, Spain) software. A bivariate random effects regression model was applied to calculate several primary outcomes, including pooled SEN, SPE, diagnostic likelihood ratio positive (DLR+), diagnostic likelihood ratio negative (DLR-), and diagnostic OR (DOR) with corresponding 95\% CIs. ${ }^{13}$ The summary receiver operator characteristic (SROC) curve was plotted and the pooled area under the curve (AUC) value was calculated. ${ }^{14}$ Cochran's $Q$ test and Higgin's $I^{2}$ statistic were used to examine heterogeneity: ${ }^{15}$ an $I^{2}>50 \%$ indicated significant heterogeneity between the studies. To explore the heterogeneity, Spearman's correlation coefficient and the $P$-value of Moss model b(1) were calculated to determine whether there was a threshold effect. Meta-regression and subgroup analysis were used to explore the potential sources of heterogeneity. Deeks' funnel plot asymmetry test was used to assess publication bias. An unequal distribution in the visual funnel plot or a $P$-value of $<0.05$ was considered to indicate statistically significant bias. ${ }^{16}$ 


\section{Results}

\section{Characteristics and quality of the included studies}

The selection procedure is presented schematically in Figure 1. We identified 877 potentially relevant articles from the online databases according to the established search criteria and the references of the determined literatures. Of these, 864 studies were excluded for the following reasons: duplicate papers $(n=147)$, review papers $(n=200)$, irrelevant contents $(n=455)$, basic research $(n=54)$, and meta-analysis $(n=8)$. Finally, 13 articles ${ }^{17-29}$ that conformed to the criteria were selected and used for statistical analysis.

\section{Study characteristics and quality assessments}

Willms et $\mathrm{al}^{26}$ studied two exosome contents, Poroyko et $\mathrm{al}^{23}$ showed nine different miRNAs in exosomes, and Fan et $\mathrm{al}^{20}$ studied four different types of populations: each content and population was considered as a separate study for our analysis. Thus, the 13 articles in this meta-analysis included 25 studies comprising 1,338 cancer cases and 1,075 controls.

The 13 articles, sorted by the year of publication (ranging from 2013 to 2018), are shown in Tables 1 and 2. Five 22,25,27-29 of the studies were conducted in China. The sample sizes of the studies ranged from 19 to 581, and all LC patients received definitive diagnosis by histopathologic examination of specimens. The paired controls comprised healthy individuals, ${ }^{18,20,22,23,26-29}$ non-LC controls, ${ }^{19,21,24,26,29}$ and a population with a relevant negative maker. ${ }^{17,18}$ Additionally, the specimen types were serum in 13 studies $^{23,26,28,29}$ and plasma in 12 studies. ${ }^{17-22,24,25,27}$ Publication languages were restricted to English and Chinese.

The outcomes of the QUADAS-2 study quality assessment are shown in Figure 2. The majority of all included studies in this meta-analysis fulfilled most items in QUADAS-2, indicating that the quality of the included studies was generally good. Table 1 lists the QUADAS score for each study.

\section{Heterogeneity}

Spearman's correlation coefficients and the $P$-value of Moss model $b(1)$ were used to evaluate heterogeneity from the threshold effect, and Cochran's $Q$ and $I^{2}$ tests were used to assess heterogeneity generated by the nonthreshold effect. In our analysis, the Spearman's correlation coefficient was $0.071(P=0.735)$ and the $P$-value of Moss model $\mathrm{b}(1)$ was

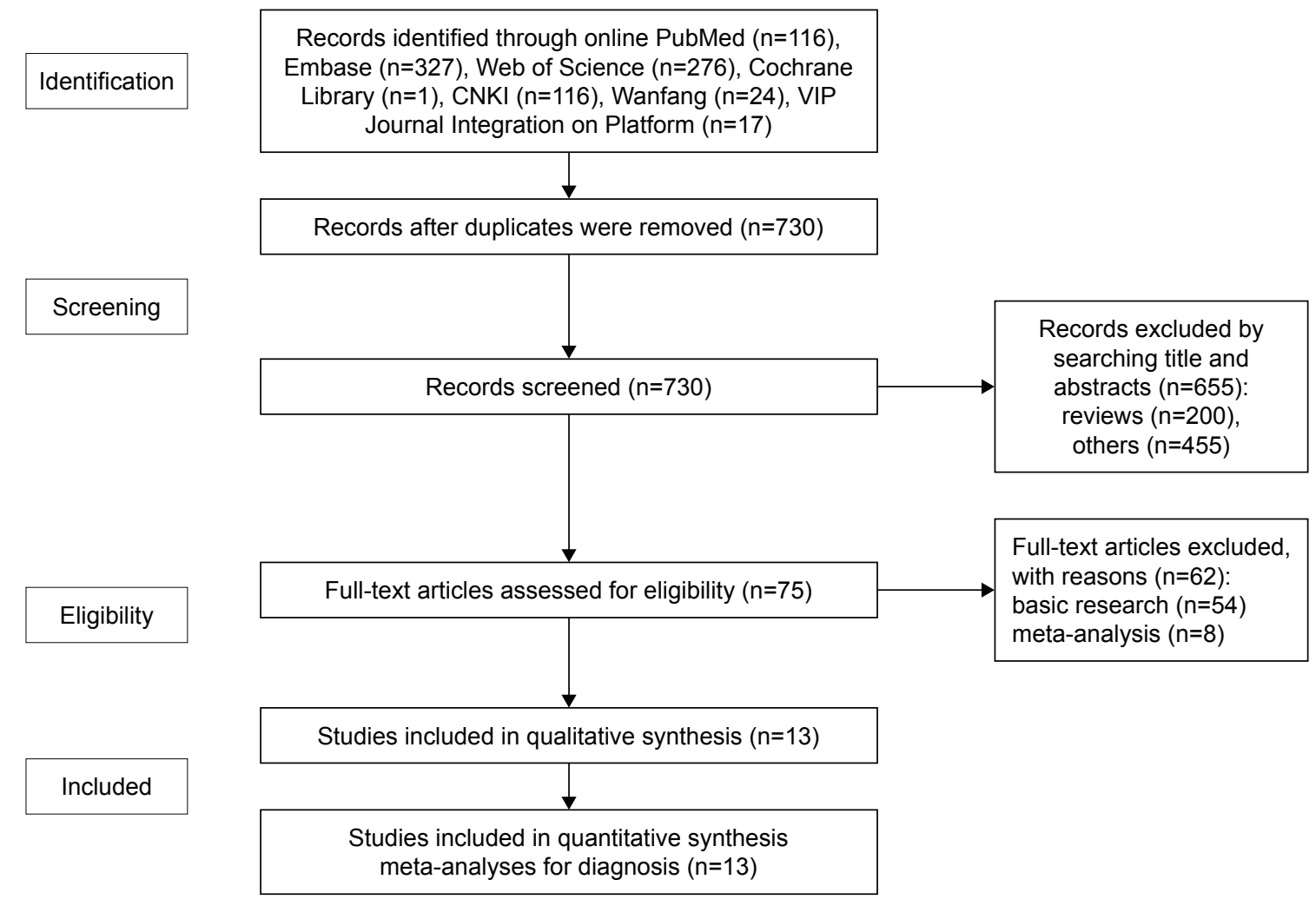

Figure I The study selection procedure for meta-analysis was conducted according to PRISMA statement. Abbreviation: CNKI, China National Knowledge Infrastructure. 


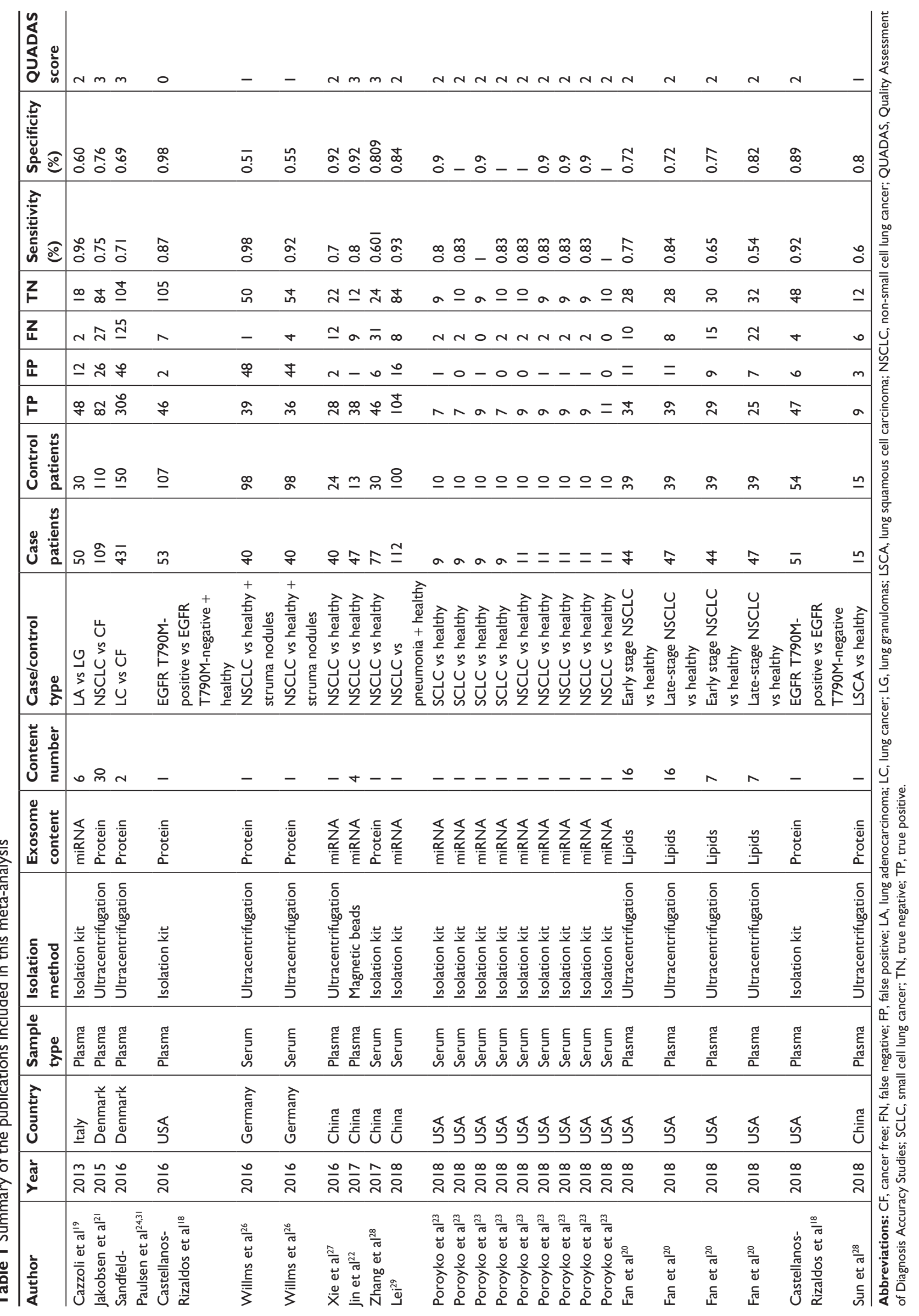


Table 2 Bibliographic information of the included articles

\begin{tabular}{|c|c|}
\hline Number & Title \\
\hline I & microRNAs derived from circulating exosomes as noninvasive biomarkers for screening and diagnosing lung cancer ${ }^{19}$ \\
\hline 2 & Exosomal proteins as potential diagnostic markers in advanced NSCLC ${ }^{21}$ \\
\hline 3 & Exosomal proteins as diagnostic biomarkers in lung cancer ${ }^{24}$ \\
\hline 4 & Plasma EGFR T790M mutation detection in NSCLC patients using a combined exosomal RNA and circulating tumor DNA qPCR assay ${ }^{18}$ \\
\hline 5 & $\begin{array}{l}\text { Tumor-associated circulating microparticles: a novel clinical tool for screening and therapy monitoring of HCC and other epithelial } \\
\text { neoplasia }^{26}\end{array}$ \\
\hline 6 & Evaluation of exosomal miRNAs from plasma as potential biomarkers for NSCLC ${ }^{27}$ \\
\hline 7 & $\begin{array}{l}\text { Evaluation of tumor-derived exosomal miRNA as potential diagnostic biomarkers for early stage NSCLC using next-generation } \\
\text { sequencing }\end{array}$ \\
\hline 8 & $\begin{array}{l}\text { Serum long non-coding RNA MALAT-I protected by exosomes is up-regulated and promotes cell proliferation and migration in } \\
\text { NSCLC }{ }^{28}\end{array}$ \\
\hline 9 & Expression and diagnostic efficacy of serum exosome miR-184 in NSCLC 29 \\
\hline 10 & Exosomal miRNAs species in the blood of small cell and NSCLC patients ${ }^{23}$ \\
\hline 11 & Exosomal lipids for classifying early and late-stage NSCLC ${ }^{20}$ \\
\hline 12 & Exosome-based detection of EGFR T790M in plasma from NSCLC patients ${ }^{17}$ \\
\hline 13 & Diagnostic value of protein markers in plasma exosomes of lung squamous cell carcinoma $a^{25}$ \\
\hline
\end{tabular}

Abbreviation: NSCLC, non-small cell lung carcinoma.

0.9483, which indicated that no threshold effects were found. The Cochran's $Q$ values for SEN and SPE were 146.95 $(P<0.05)$ and $157.49(P<0.05)$, respectively. The $I^{2}$ values for SEN and SPE were 83.67 (95\% CI: 78.03-89.31) and 84.76 (95\% CI: 79.60-89.92), which indicated the existence of significant heterogeneity.

\section{Diagnostic performance}

The forest plots of pooled SEN and SPE are shown in Figure 3. The pooled SEN of the 25 studies was 0.82 (95\% CI: $0.76-0.87$ ) and the pooled SPE was 0.84 (95\% CI: 0.77-0.89). The forest plots of DLR+ and DLR- are shown in Figure 4. The pooled DLR+ and DLR- were 5.27 (95\% CI: 3.58-7.75) and 0.21 (95\% CI: 0.15-0.29), respectively. Figure 5 shows the DOR was 25.14 (95\% CI: 14.25-44.33), and Figure 6 shows that the AUC was 0.90 (95\% CI: 0.87-0.92). These findings demonstrate that liquid exosomes have an overall high diagnostic efficacy for LC.

\section{Meta-regression and subgroup analysis}

Meta-regression and subgroup analysis were performed to explore potential heterogeneity. Research country (China or not), isolation method 1 (isolation kit or not), isolation method 2 (ultracentrifugation or not), isolation method 3 (magnetic beads or not), sample type (plasma or not), type of exosome content 1 (miRNA or not), type of exosome content 2 (protein or not), type of exosome content 3 (lipids or not), content number (many or not), patient size $(<50$ or not), control size ( $<50$ or not), and QUADAS score $(<2$ or not) were used as covariates in the meta-regression. As shown in Figure 7, research country, sample type, isolation method 2, type of exosome content 2, type of exosome content 3 , content number, patient size, and control size had $P$-values $<0.05$, indicating that these reasons were likely the sources of heterogeneity in SEN. Furthermore, sample type, isolation method 2, type of exosome content 2, type of exosome content 3, content number, and QUADAS score had $P$-values $<0.05$, meaning that these factors were likely the sources of heterogeneity in SPE.

Subgroup analysis was performed using the platform of Meta-disc 1.4 software. Seven characteristics (sample type, experimental method used for exosome isolation, type of exosome content, content number, patient size, control size, and type of LC) were considered. As shown in Table 3, we found differences between plasma-based and serum-based analyses: SEN (0.75 vs 0.84), DLR+ (3.61 vs 4.75), DOR (15.57 vs 31.29$)$, and AUC (0.8696 vs 0.9172) were significantly higher in serum, providing additional support for the use of serum to evaluate relatively reliable diagnostic biomarkers. Using different experimental methods to isolate exosomes showed different results; isolation kit seemed to be the optimal method because of its high SEN, SPE, and AUC, which were $0.85,0.89$, and 0.9413 , respectively. Since there was only one study that used the magnetic beads method, the results shown in Table 3 came directly from the study. In the meta-analysis based on miRNA, one index in exosome, patient size of 50 or higher, and control size of 50 or higher had higher AUC values of 0.9404, 0.9252, 0.9101 , and 0.9241 , respectively. Moreover, the analysis of the type of LC suggested that liquid exosomes had a strong ability to diagnose small cell LC with SEN, SPE, and AUC of 0.83 (95\% CI: 0.67-0.94), 0.95 (95\% CI: 0.83-0.99) and 0.9518 , respectively. 


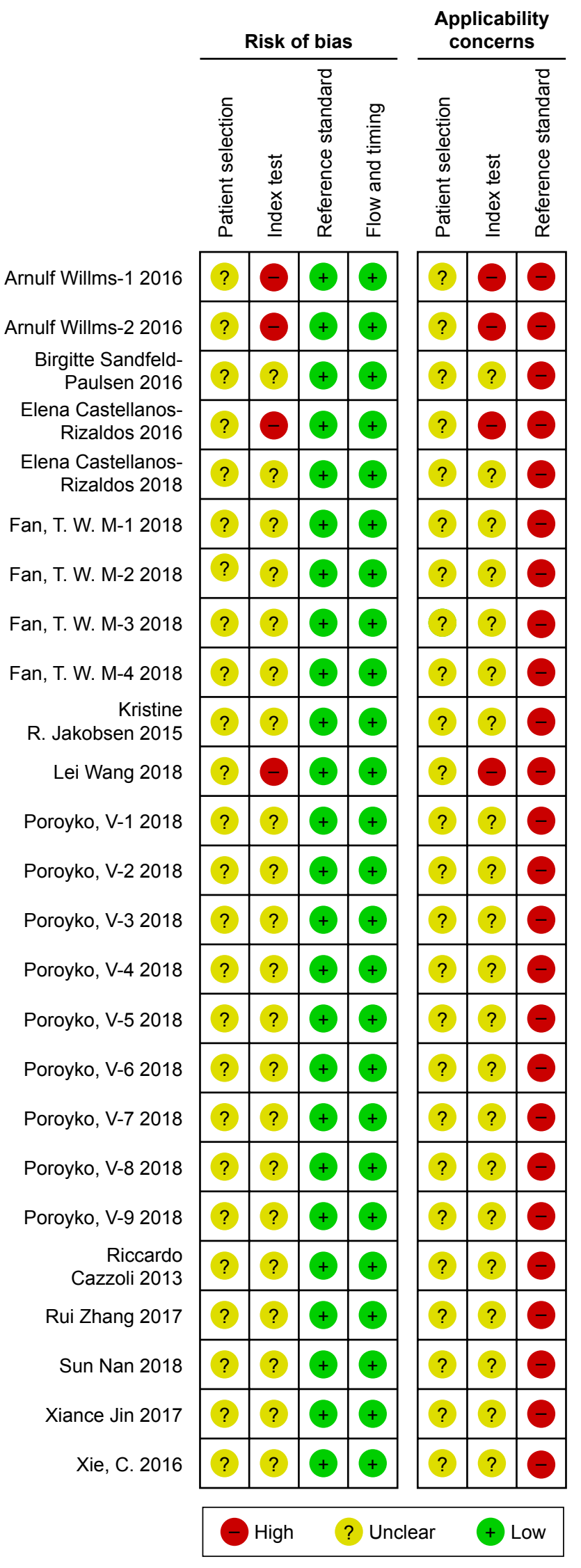

Figure 2 Overall quality assessment of the included articles using QUADAS-2 tool. Abbreviation: QUADAS, Quality Assessment of Diagnostic Accuracy Studies.

\section{Publication bias}

Deeks' funnel plot asymmetry test was performed to evaluate publication bias. As shown in Figure 8, the $P$-value was 0.10, which is $>0.05$, indicating that no significant publication bias was found among these studies.

\section{Discussion}

Screening for LC is particularly important due to its high incidence and low survival rates. ${ }^{1,30}$ However, affordable, repeatable, stable, and precise detection methods are not available. ${ }^{22}$ Although liquid exosomes might have a high diagnostic value, they are less reliable and less consistent than other diagnostic tools for the detection of different sample types and contents in exosomes. ${ }^{19,21,28,31}$ In order to examine the reported diagnostic efficacy of liquid exosomes in LC and to evaluate whether they could be rated as additional molecular markers to aid in LC diagnosis, we conducted a meta-analysis to define the overall diagnostic accuracy of liquid exosomes in LC.

In all, 25 studies conducted from 2013 to 2018 that involved a total of 2,413 patients ( $1,338 \mathrm{LC}$ patients and 1,075 controls) were included in this meta-analysis. AUC is widely recognized as a useful index for evaluating the accuracy of diagnostic tests. If AUC is $>0.9$, the diagnostic value is considered to be high. The ideal position, which is indicative of a perfect test, is near the upper-left corner in an SROC curve. In our analysis, the SROC curve had an AUC of 0.90, which indicated that liquid exosomes could be a powerful biomarker for LC detection. The pooled SEN and SPE values for all the studies were 0.82 and 0.84 , respectively. DOR, a comprehensive evaluation index in diagnostic tests, was used to investigate multiple relationships between chances of obtaining positive and negative results. The pooled DOR was 25.14 , indicating that liquid exosomes could be a useful diagnostic biomarker for LC. Overall, our results from liquid exosomes in LC detection demonstrate that, as diagnostic biomarkers, they are promising candidates with high overall accuracy.

We used meta-regression and subgroup analysis to explore the heterogeneity among studies. First, we performed meta-regression, and we considered 12 covariates (research country, sample type, using isolation kit to isolate exosomes, using ultracentrifugation to isolate exosomes, using magnetic beads to isolate exosomes, miRNA as the exosome content, protein as the exosome content, lipids as the exosome content, content number, patient size, control size, and QUADAS-2 score). On the basis of our results, different sample types, experimental methods used for exosome isolation, and content number were responsible for the homogeneity of both 


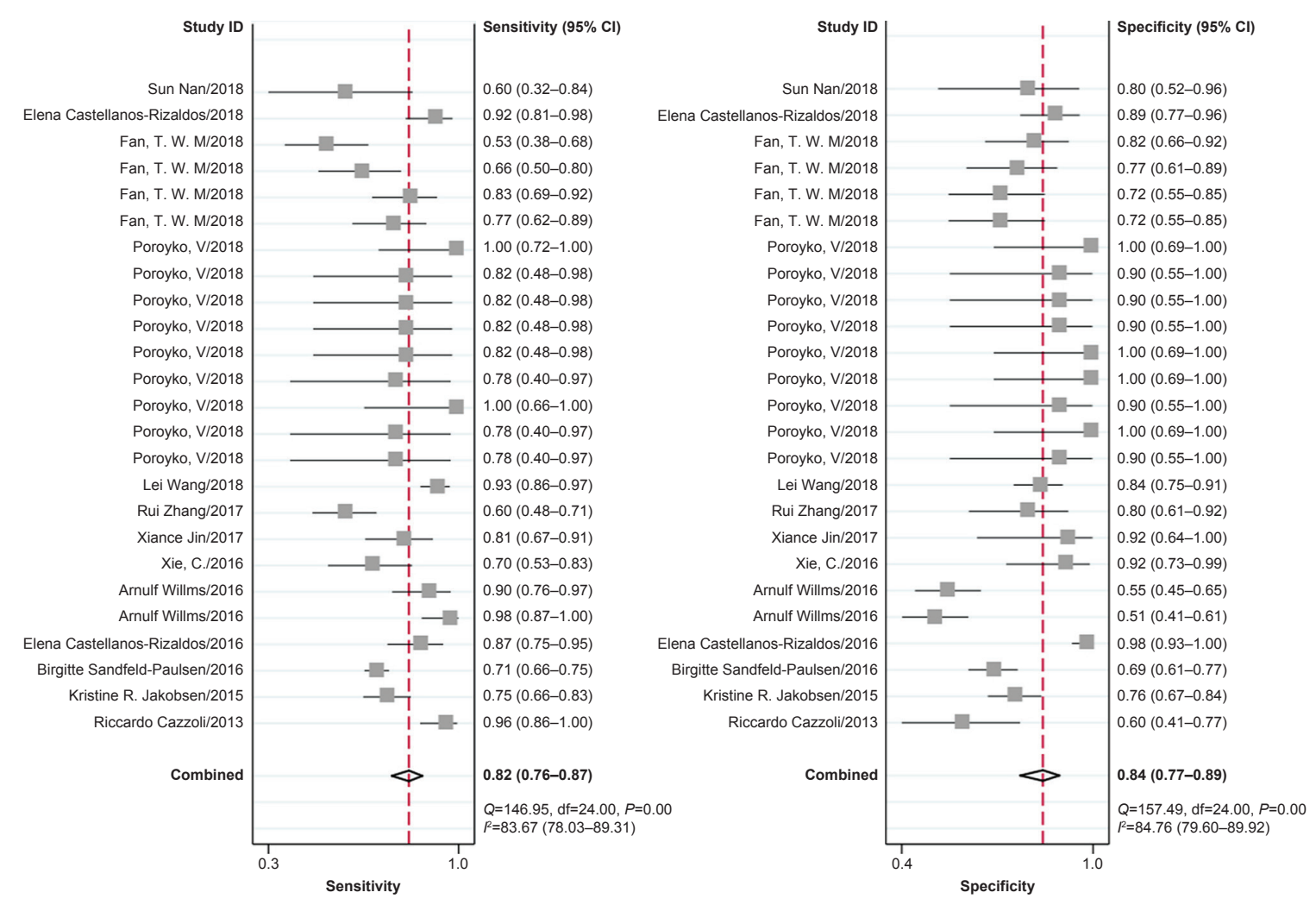

Figure 3 Forest plot of sensitivity and specificity of liquid exosomes for the diagnosis of LC.

Abbreviations: $\mathrm{df}$, degrees of freedom; DLR, diagnostic likelihood ratio; LC, lung cancer.

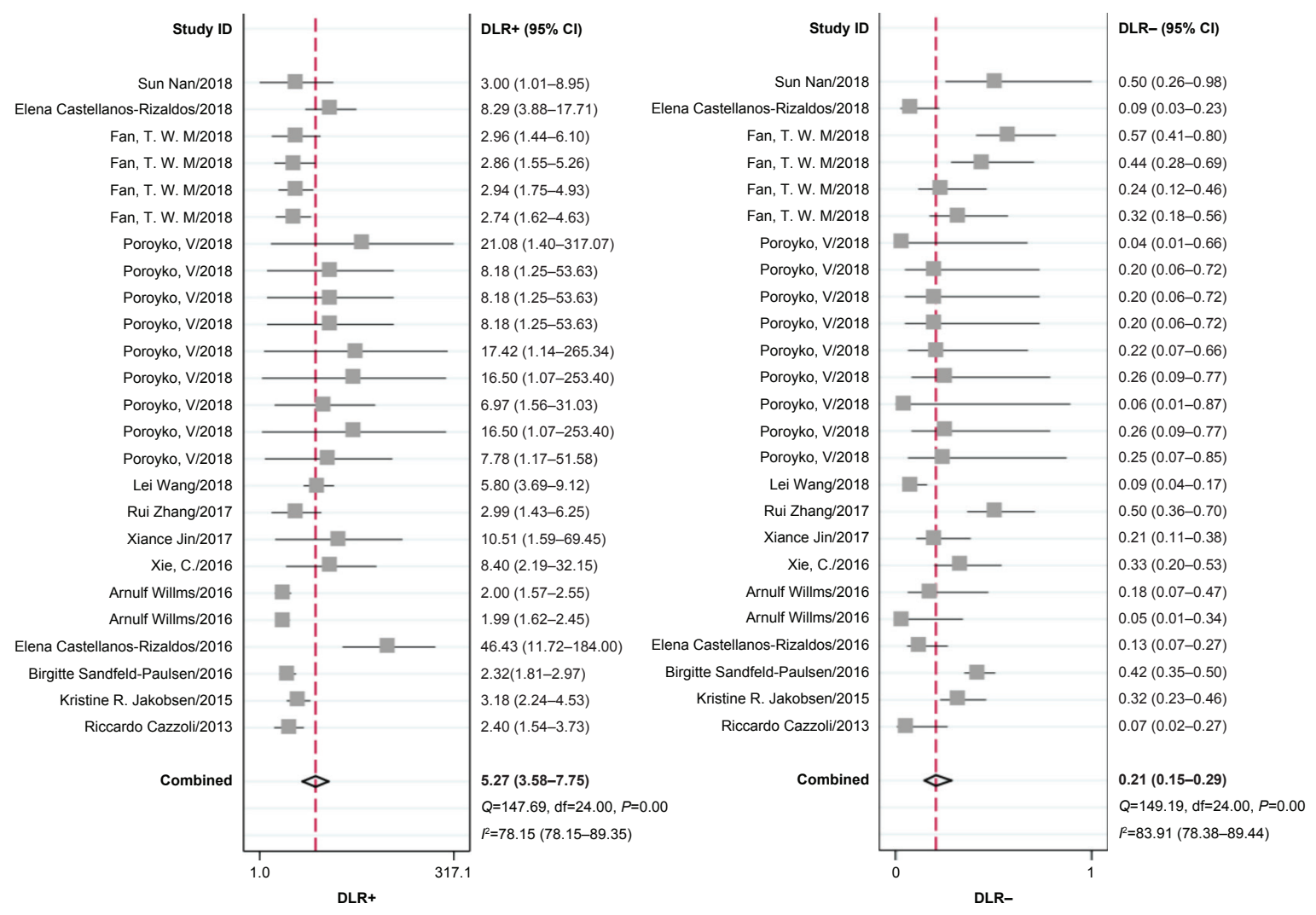

Figure 4 Forest plot of DLR+ and DLR- of liquid exosomes for the diagnosis of LC.

Abbreviations: df, degrees of freedom; DLR, diagnostic likelihood ratio; LC, lung cancer. 


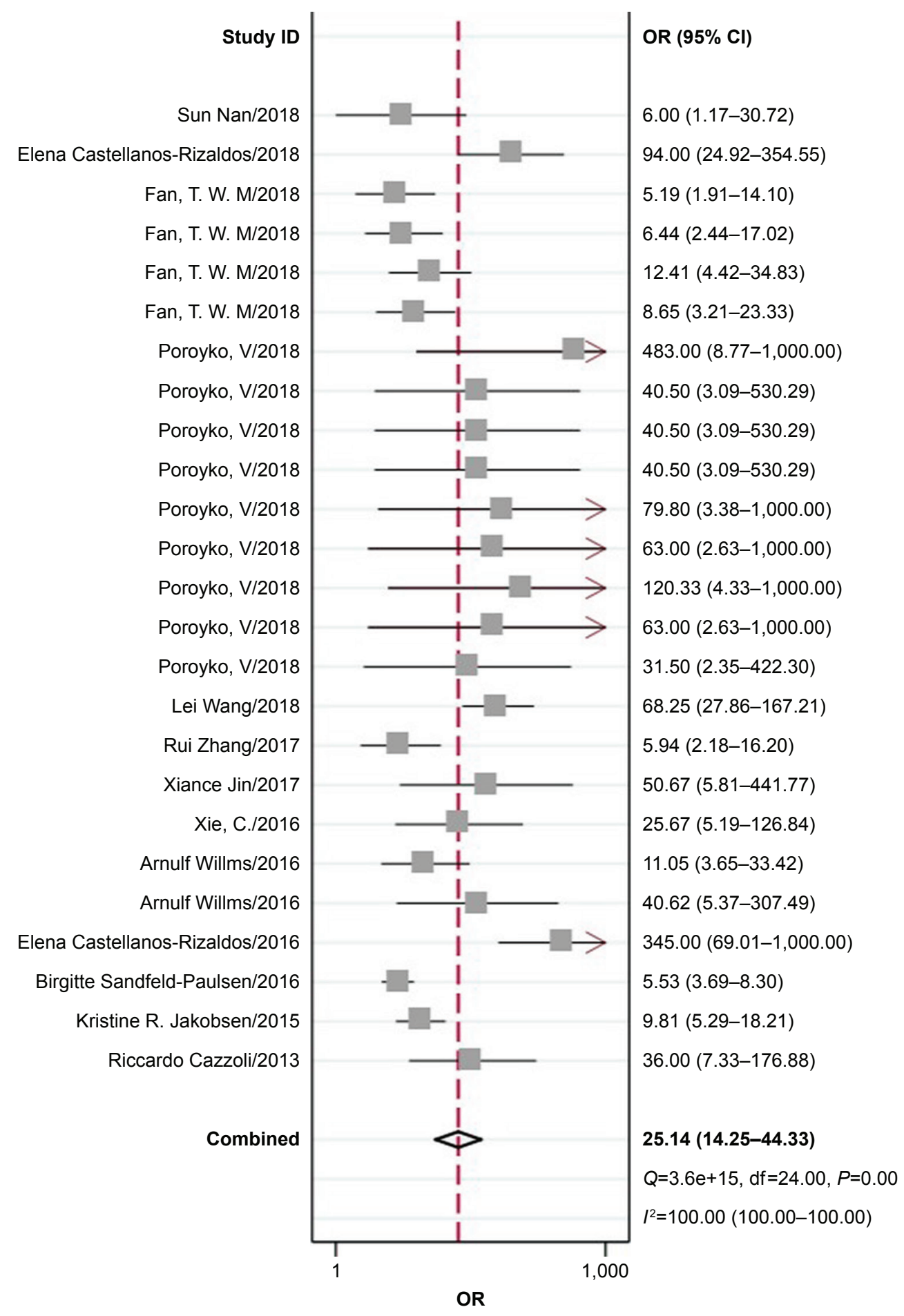

Figure 5 Forest plot of DOR of liquid exosomes for the diagnosis of LC.

Abbreviations: df, degrees of freedom; DOR, diagnostic OR; LC, lung cancer.

SEN and SPE. Second, we used subgroup analysis to further explore the heterogeneity. Seven characteristics (sample type, experimental method used for exosome isolation, type of exosome content, content number, patient size, control size, and type of LC) were considered.
Although pooled SEN, DLR+, DOR, and AUC of serum were significantly higher than the same measurements in plasma, the SPE of plasma was higher than the SPE of serum. It is possible that the sample differences caused the differences in diagnostic performance of the liquid exosome 


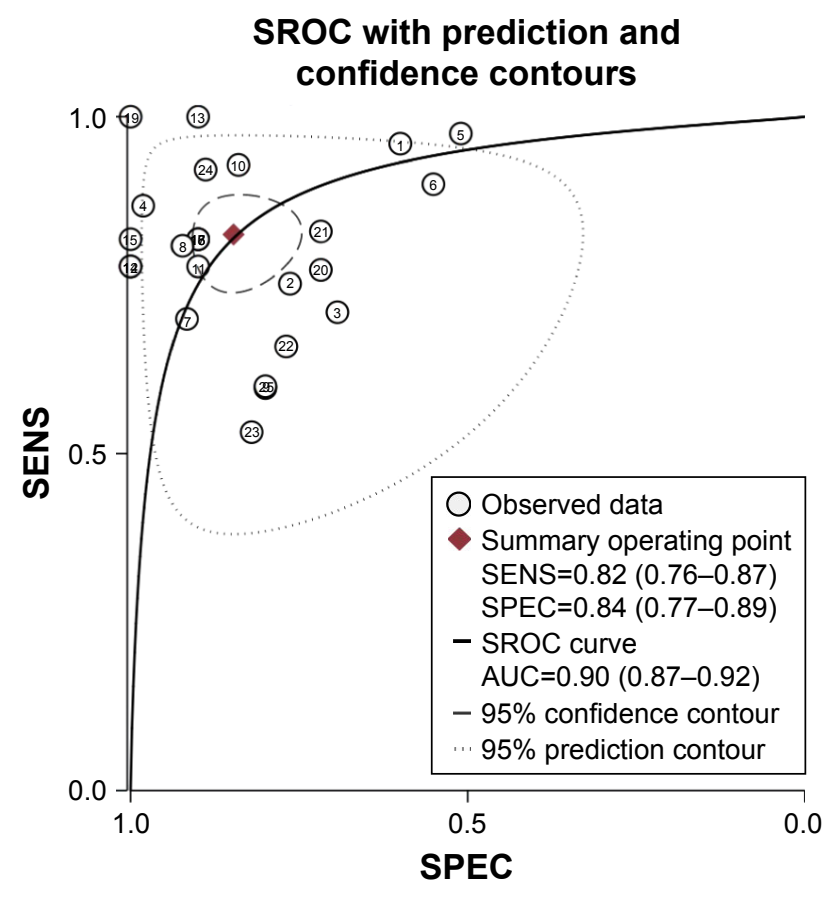

Figure 6 The SROC of the liquid exosomes test for the diagnosis of LC. Abbreviations: AUC, area under the curve; LC, lung cancer; SENS, sensitivity; SPEC, specificity; SROC, summary receiver operator characteristic.

detection. McDonald et al ${ }^{32}$ demonstrated that, compared to serum, miRNA concentrations were higher in plasma, and sample types were critical factors in investigating the utility of circulating miRNAs as biomarkers. However, the origin of source-related differences in exosomes is still unclear and might be explained by an unknown mechanism. Additionally, extracellular vesicles released by activated platelets may affect the purity level when isolating exosomes from the serum..$^{33}$ The presence of anticoagulant in plasma may also affect subsequent experimental results. There is no consensus regarding whether plasma or serum is preferable as a sample. The results of our analysis suggested that we should explore and study how the different sample types influence exosome levels, and large-scale investigations are needed to determine whether the source-related differences truly exist or not.

The studies in this meta-analysis used three different methods to isolate exosomes. Evidence supports that the isolation kit method obtains superior diagnostic values, but we cannot verify this theory. Since most included studies used this method, the sample size of the isolation kit method is larger than that of other methods. Perhaps this is the reason why the isolation kit method showed better results.

Clearly, the SEN, SPE, and AUC are high when the exosome content is miRNA. MiRNAs are short, noncoding
RNAs, and a single miRNA can regulate the expressions of thousands of genes and participate in the regulation of the entire cell cycle. Since the circulating miRNAs in serum was first reported,${ }^{34}$ growing interest has been focused on the feasibility of circulating miRNAs as potential biomarkers in $\mathrm{LC}$ diagnosis. They have a promising future as a novel class diagnostic due to their remarkable stability, relative ease of detectability, and convenience for measuring its SEN and SPE. ${ }^{35}$ The diagnostic value and mechanism of miRNAs in exosomes require more in-depth research.

When the content number equaled one, the SEN, SPE, and AUC were better than they were for multiple indicators. It is widely accepted that using a single index in exosomes as disease fingerprints is simpler and more straightforward than comprehensively detecting panels, which was proved in our analysis. Because most included studies were detecting one index (17 vs 8 ), the sample size of the population studying one index is larger than the sample size of other index detection methods. This may be why one-index detection showed better results.

This meta-analysis has several limitations that require consideration. First, we may have exaggerated the relative risk because the pooled results are based on only 13 included articles with relatively small sample sizes. This may influence a variety of confounding factors for the final results. Further, samples were tested at different points in time. Although miRNAs are stable in serum and plasma, results may have been altered due to testing at different times and under varied conditions. There is no consensus regarding the internal control of normalization for exosome miRNA quantification. In addition, although almost all the control groups were composed of cancer-free patients, inclusion criteria and baseline data for these groups varied among different studies. Unfortunately, there are no qualified articles regarding other kinds of sample types. The incidence of LC in males and females may vary, and these factors were not considered in our meta-analysis. Second, any relevant articles that have not yet been published online might be missing. We extracted data from English and Chinese language studies only, and this could also have influenced our results. Third, due to the significant heterogeneity, we could only roughly estimate the value of liquid exosomes in LC diagnosis. In order to fully explore their potential clinical value, more research is needed to assess the diagnostic accuracy of liquid exosomes in different specimens, types, and stages of LC. Finally, publication bias is possible, even if a comprehensive and systematic search of literature was completed. 


\section{Univariable meta-regression and subgroup analyses}
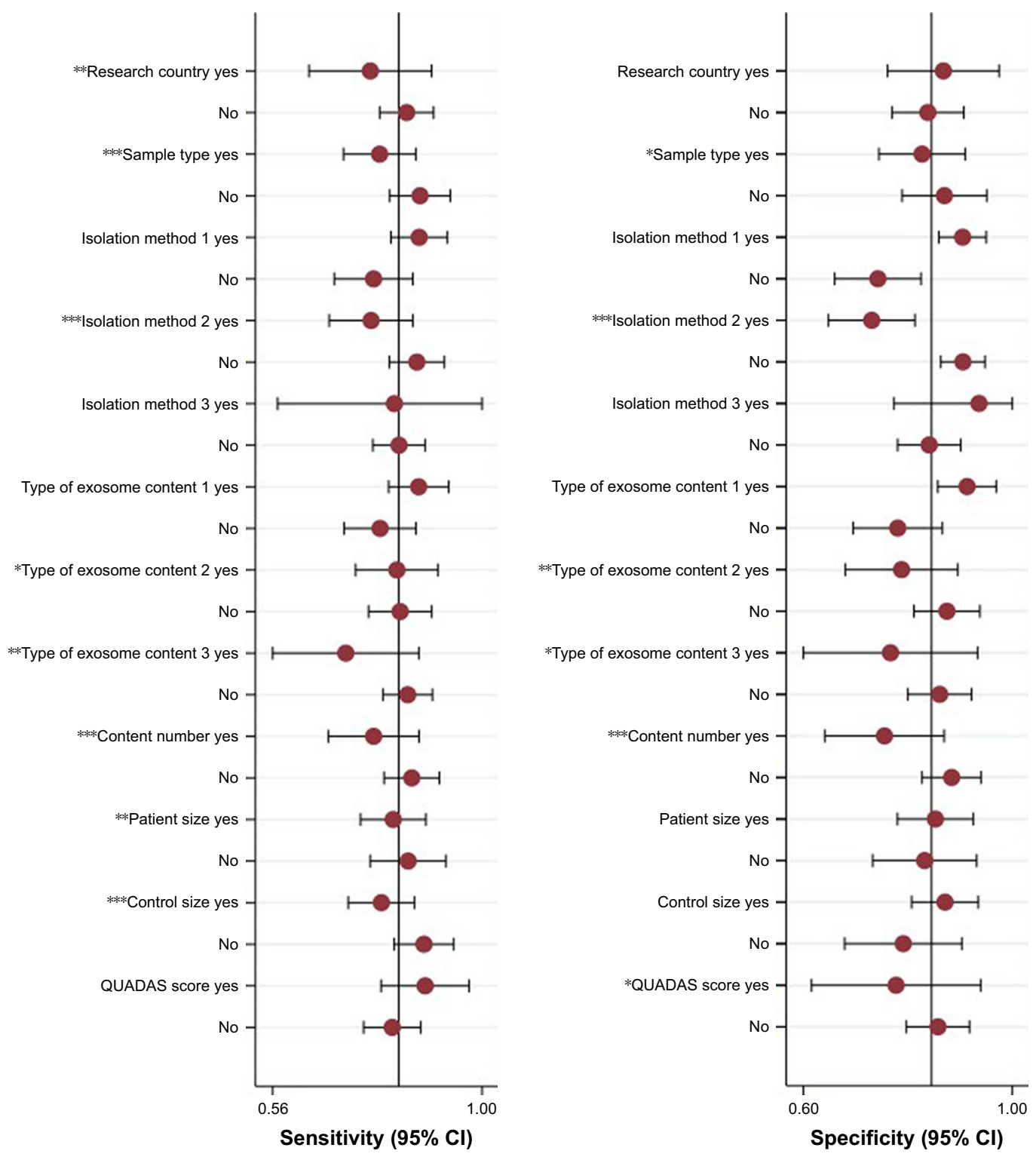

Figure 7 Forest plot of covariates' meta-regression.

Note: $* P<0.05, * * P<0.01$, $* * * P<0.001$.

Table 3 Summary results of subgroup analysis for liquid exosomes in the diagnosis of LC

\begin{tabular}{|c|c|c|c|c|c|c|c|}
\hline Subgroups & $\begin{array}{l}\text { Number } \\
\text { of studies }\end{array}$ & SEN (95\% Cl) & SPE $(95 \% \mathrm{Cl})$ & $\begin{array}{l}\text { DLR+ } \\
(95 \% \mathrm{Cl})\end{array}$ & \begin{tabular}{|l|} 
DLR- \\
$(95 \% \mathrm{Cl})$
\end{tabular} & DOR $(95 \% \mathrm{Cl})$ & AUC \\
\hline \multicolumn{8}{|l|}{ Sample type } \\
\hline Plasma & 12 & $0.75(0.72-0.77)$ & $0.79(0.76-0.82)$ & $3.61(2.64-4.93)$ & $0.30(0.22-0.39)$ & I $5.57(8.40-28.85)$ & 0.8696 \\
\hline Serum & 13 & $0.84(0.80-0.88)$ & $0.71(0.67-0.76)$ & $4.75(2.79-8.07)$ & $0.18(0.10-0.33)$ & $31.29(15.30-63.96)$ & 0.9172 \\
\hline \multicolumn{8}{|l|}{ Isolation method } \\
\hline Isolation kit & 14 & $0.85(0.8 \mathrm{I}-0.88)$ & $0.89(0.85-0.91)$ & $7.10(4.24-11.88)$ & $0.17(0.10-0.29)$ & $53.37(25.16-113.23)$ & 0.9413 \\
\hline Ultracentrifugation & 10 & $0.73(0.70-0.76)$ & $0.68(0.64-0.72)$ & $2.47(2.09-2.93)$ & $0.37(0.29-0.46)$ & $7.91(5.86-10.67)$ & 0.8016 \\
\hline Magnetic beads & 1 & $0.80(-)$ & $0.92(-)$ & $10.00(-)$ & $0.22(-)$ & $45.45(-)$ & 0.8990 \\
\hline
\end{tabular}


Table 3 (Continued)

\begin{tabular}{|c|c|c|c|c|c|c|c|}
\hline Subgroups & $\begin{array}{l}\text { Number } \\
\text { of studies }\end{array}$ & SEN $(95 \% \mathrm{Cl})$ & SPE (95\% Cl) & $\begin{array}{l}\text { DLR+ } \\
(95 \% \mathrm{Cl})\end{array}$ & $\begin{array}{l}\text { DLR- } \\
(95 \% \mathrm{Cl})\end{array}$ & DOR (95\% Cl) & AUC \\
\hline \multicolumn{8}{|l|}{ Type of exosome content } \\
\hline miRNA & 13 & $0.87(0.83-0.90)$ & $0.86(0.8 \mathrm{I}-0.90)$ & $6.19(3.85-9.96)$ & $0.19(0.13-0.27)$ & $52.30(30.16-90.72)$ & 0.9404 \\
\hline Protein & 8 & $0.75(0.72-0.78)$ & $0.73(0.69-0.76)$ & $3.26(2.22-4.79)$ & $0.27(0.17-0.4 I)$ & $17.29(7.45-40.10)$ & 0.8818 \\
\hline Lipids & 4 & $0.70(0.63-0.76)$ & $0.76(0.68-0.82)$ & $2.86(2.14-3.82)$ & $0.40(0.27-0.58)$ & $7.67(4.66-12.63)$ & 0.8102 \\
\hline \multicolumn{8}{|l|}{ Content number } \\
\hline Many & 8 & $0.73(0.70-0.76)$ & $0.73(0.69-0.77)$ & $2.66(2.27-3.10)$ & $0.34(0.26-0.45)$ & $8.52(5.70-12.73)$ & 0.8052 \\
\hline One & 17 & $0.83(0.80-0.86)$ & $0.79(0.75-0.82)$ & $6.22(3.60-10.75)$ & $0.20(0 . \mid 3-0.31)$ & 37.76 (19.19-74.28) & 0.9252 \\
\hline \multicolumn{8}{|l|}{ Patient size } \\
\hline$<50$ & 18 & $0.78(0.74-0.82)$ & $0.7 \mathrm{I}(0.67-0.75)$ & $3.63(2.58-5.10)$ & $0.29(0.22-0.38)$ & $14.65(9.33-23.01)$ & 0.8642 \\
\hline$\geq 50$ & 7 & $0.77(0.74-0.80)$ & $0.80(0.77-0.84)$ & $4.28(2.69-6.82)$ & $0.20(0.11-0.35)$ & $26.99(9.6 I-75.82)$ & 0.9101 \\
\hline \multicolumn{8}{|l|}{ Control size } \\
\hline$<50$ & 18 & $0.74(0.70-0.78)$ & $0.81(0.77-0.85)$ & $3.40(2.67-4.34)$ & $0.31(0.24-0.40)$ & $14.42(9.00-23.12)$ & 0.8657 \\
\hline$\geq 50$ & 7 & $0.79(0.76-0.82)$ & $0.74(0.70-0.77)$ & $3.78(2.40-5.93)$ & $0.17(0.09-0.31)$ & $30.01(10.50-85.74)$ & $0.924 \mid$ \\
\hline \multicolumn{8}{|l|}{ Type of LC } \\
\hline NSCLC & 18 & $0.79(0.76-0.82)$ & $0.73(0.69-0.76)$ & $3.25(2.49-4.25)$ & $0.26(0.19-0.36)$ & $15.48(9.73-24.64)$ & 0.8627 \\
\hline SCLC & 4 & $0.83(0.67-0.94)$ & $0.95(0.83-0.99)$ & $9.06(3.33-24.69)$ & $0.24(0.13-0.45)$ & $56.92(12.54-258.40)$ & 0.9518 \\
\hline Others & 3 & $0.75(0.71-0.78)$ & $0.83(0.78-0.87)$ & 8.55 (1.75-4I.77) & $0.18(0.05-0.62)$ & $52.16(3.36-809.53)$ & 0.9139 \\
\hline
\end{tabular}

Abbreviations: AUC, area under the curve; DLR+, diagnostic likelihood ratio positive; DLR-, diagnostic likelihood ratio negative; LC, lung cancer; NSCLC, non-small cell lung cancer; SCLC, small cell lung cancer; SEN, sensitivity; SPE, specificity.

\section{Conclusion}

Overall, our quantitative meta-analysis found that liquid exosomes had high diagnostic value for $\mathrm{LC}$, and we confirmed the diagnostic efficacy of liquid exosome signatures as promising biomarkers for LC detection. However, sample type, experimental method used for exosome isolation, type of exosome content, content number, sample size, and type of LC were associated

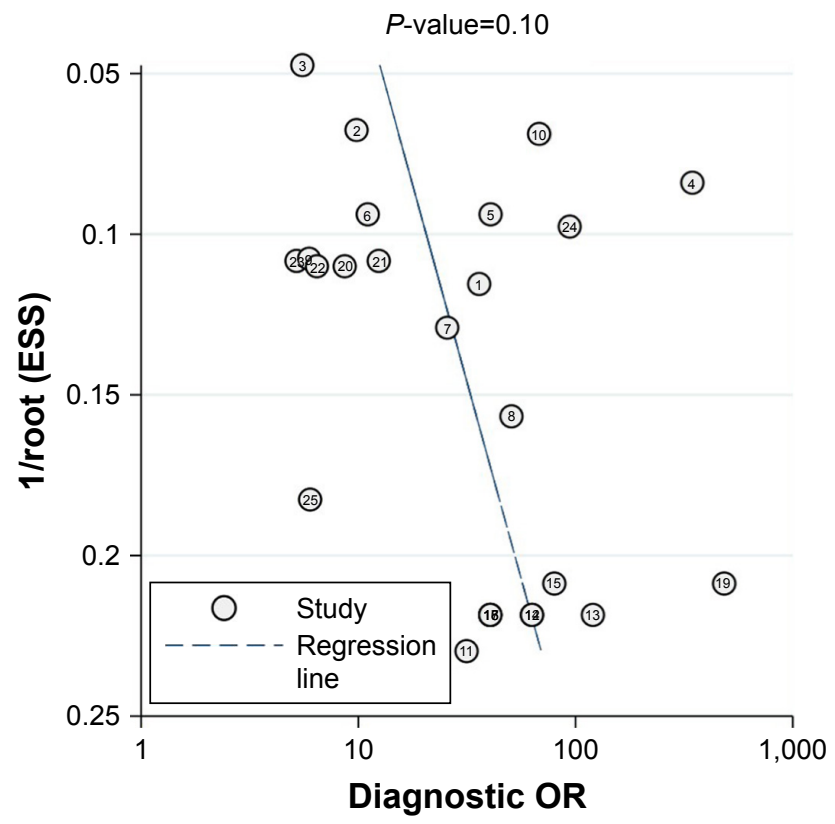

Figure 8 Deeks' funnel plot asymmetry test for assessing publication bias. Abbreviation: ESS, effective sample size. with different results. Therefore, further studies on the diagnostic performance of liquid exosomes in LC are warranted.

\section{Acknowledgment}

This study was supported by the foundation project of High-Level Health Technology Talents in the Beijing Health System (No 2015-3-097), Key Tasks of the Beijing Municipal Party Committee, the Municipal Government and Pre-launch of District Government Emergency Projects (No. z151100002115049). We also thank BioMed Proofreading LLC for its linguistic assistance during the preparation of this manuscript.

\section{Disclosure}

The authors report no conflicts of interest in this work.

\section{References}

1. Bray F, Ferlay J, Soerjomataram I, Siegel RL, Torre LA, Jemal A. Global cancer statistics 2018: GLOBOCAN estimates of incidence and mortality worldwide for 36 cancers in 185 countries. CA Cancer J Clin. 2018; 68(6):394-424.

2. Diaz LA, Bardelli A. Liquid biopsies: genotyping circulating tumor DNA. J Clin Oncol. 2014;32(6):579-586.

3. Tan DS, Camilleri-Broët S, Tan EH, et al. Intertumor heterogeneity of non-small-cell lung carcinomas revealed by multiplexed mutation profiling and integrative genomics. Int J Cancer. 2014;135(5):1092-1100.

4. Wille MM, Dirksen A, Ashraf H, et al. Results of the randomized Danish lung cancer screening trial with focus on high-risk profiling. Am J Respir Crit Care Med. 2016;193(5):542-551.

5. Tanoue LT, Tanner NT, Gould MK, Silvestri GA. Lung cancer screening. Am J Respir Crit Care Med. 2015;191(1):19-33. 
6. Zheng H, Zhan Y, Liu S, et al. The roles of tumor-derived exosomes in non-small cell lung cancer and their clinical implications. J Exp Clin Cancer Res. 2018;37(1):226.

7. Vanni I, Alama A, Grossi F, Dal Bello MG, Coco S. Exosomes: a new horizon in lung cancer. Drug Discov Today. 2017;22(6):927-936.

8. Rodríguez M, Silva J, López-Alfonso A, et al. Different exosome cargo from plasma/bronchoalveolar lavage in non-small-cell lung cancer. Genes Chromosomes Cancer. 2014;53(9):713-724.

9. Taverna S, Giallombardo M, Gil-Bazo I, et al. Exosomes isolation and characterization in serum is feasible in non-small cell lung cancer patients: critical analysis of evidence and potential role in clinical practice. Oncotarget. 2016;7(19):28748-28760.

10. Zou $\mathrm{H}, \mathrm{Wu} \mathrm{H}, \mathrm{Xu} \mathrm{C}$. Research progress of exosomes in lung cancer diagnosis and treatment. Zhongguo Fei Ai Za Zhi. 2016;19(11):778-783.

11. Moher D, Liberati A, Tetzlaff J, Altman DG; PRISMA Group. Preferred reporting items for systematic reviews and meta-analyses: the PRISMA statement. Int J Surg. 2010;8(5):336-341.

12. Whiting PF, Rutjes AW, Westwood ME, et al. QUADAS-2: a revised tool for the quality assessment of diagnostic accuracy studies. Ann Intern Med. 2011;155(8):529-536.

13. Reitsma JB, Glas AS, Rutjes AW, Scholten RJ, Bossuyt PM, Zwinderman AH. Bivariate analysis of sensitivity and specificity produces informative summary measures in diagnostic reviews. J Clin Epidemiol. 2005;58(10):982-990.

14. Hamza TH, Arends LR, van Houwelingen HC, Stijnen T. Multivariate random effects meta-analysis of diagnostic tests with multiple thresholds. BMC Med Res Methodol. 2009;9(1):73.

15. Higgins JP, Thompson SG, Deeks JJ, Altman DG. Measuring inconsistency in meta-analyses. BMJ. 2003;327(7414):557-560.

16. Deeks JJ, Macaskill P, Irwig L. The performance of tests of publication bias and other sample size effects in systematic reviews of diagnostic test accuracy was assessed. J Clin Epidemiol. 2005;58(9):882-893.

17. Castellanos E. Exosome-based detection of EGFR T790M in plasma from non-small cell lung cancer patients. Mol Cancer Ther. 2018;17(1).

18. Castellanos-Rizaldos E, Grimm DG, Tadigotla V, et al. Plasma EGFR T790M mutation detection in NSCLC patients using a combined exosomal RNA and circulating tumor DNA qPCR assay. Eur J Cancer. 2016;69:S3.

19. Cazzoli R, Buttitta F, Di Nicola M, et al. microRNAs derived from circulating exosomes as noninvasive biomarkers for screening and diagnosing lung cancer. J Thorac Oncol. 2013;8(9):1156-1162.

20. Fan TWM, Zhang X, Wang C, et al. Exosomal lipids for classifying early and late stage non-small cell lung cancer. Anal Chim Acta. 2018;1037: 256-264.

21. Jakobsen KR, Paulsen BS, Bæk R, Varming K, Sorensen BS, Jørgensen MM. Exosomal proteins as potential diagnostic markers in advanced non-small cell lung carcinoma. J Extracell Vesicles. 2015; 4(1):26659.
22. Jin $\mathrm{X}$, Chen $\mathrm{Y}$, Chen $\mathrm{H}$, et al. Evaluation of tumor-derived exosomal miRNA as potential diagnostic biomarkers for early-stage non-small cell lung cancer using next-generation sequencing. Clin Cancer Res. 2017;23(17):5311-5319.

23. Poroyko V, Mirzapoiazova T, Nam A, et al. Exosomal miRNAs species in the blood of small cell and non-small cell lung cancer patients. Oncotarget. 2018;9(28):19793-19806.

24. Sandfeld-Paulsen B, Jakobsen KR, Bæk R, et al. Exosomal proteins as diagnostic biomarkers in lung cancer. J Thorac Oncol. 2016;11(10): 1701-1710.

25. Sun N, Sun SG, Lu ZL, He J. [Diagnostic value of protein markers in plasma exosomes of lung squamous cell carcinoma]. Zhonghua Zhong Liu Za Zhi. 2018;40(6):418-421. Chinese.

26. Willms A, Müller C, Julich H, et al. Tumor-associated circulating microparticles: a novel clinical tool for screening and therapy monitoring of HCC and other epithelial neoplasia. J Hepatol. 2016;64(2):S336-S337.

27. Xie C, Jin X, Fei Z, Su H, Zhao L. Evaluation of exosomal miRNAs from plasma as potential biomarkers for non-small-cell lung cancer. J Clin Oncol. 2016;34(15_suppl):e20004.

28. Zhang R, Xia Y, Wang Z, et al. Serum long non coding RNA MALAT-1 protected by exosomes is up-regulated and promotes cell proliferation and migration in non-small cell lung cancer. Biochem Biophys Res Commun. 2017;490(2):406-414.

29. Lei W. Expression and diagnostic efficacy of serum exosome miR-184 in non-small cell lung cancer. J Lanzhou Univ. 2018;03: 31-36.

30. Siegel RL, Miller KD, Jemal A. Cancer statistics, 2016. CA Cancer J Clin. 2016;66(1):7-30.

31. Sandfeld-Paulsen B, Jakobsen KR, Bæk R, et al. Exosomal proteins as diagnostic biomarkers in lung cancer. J Thorac Oncol. 2016;11(10): 1701-1710.

32. McDonald JS, Milosevic D, Reddi HV, Grebe SK, AlgecirasSchimnich A. Analysis of circulating microRNA: preanalytical and analytical challenges. Clin Chem. 2011;57(6):833-840.

33. Brisson AR, Tan S, Linares R, Gounou C, Arraud N. Extracellular vesicles from activated platelets: a semiquantitative cryo-electron microscopy and immuno-gold labeling study. Platelets. 2017;28(3):263-271.

34. Lawrie CH, Gal S, Dunlop HM, et al. Detection of elevated levels of tumour-associated microRNAs in serum of patients with diffuse large B-cell lymphoma. Br J Haematol. 2008;141(5):672-675.

35. Redova M, Sana J, Slaby O. Circulating miRNAs as new blood-based biomarkers for solid cancers. Future Oncol. 2013;9(3):387-402.
OncoTargets and Therapy

\section{Publish your work in this journal}

OncoTargets and Therapy is an international, peer-reviewed, open access journal focusing on the pathological basis of all cancers, potential targets for therapy and treatment protocols employed to improve the management of cancer patients. The journal also focuses on the impact of management programs and new therapeutic agents and protocols on

\section{Dovepress}

patient perspectives such as quality of life, adherence and satisfaction The manuscript management system is completely online and includes a very quick and fair peer-review system, which is all easy to use. Visit http://www.dovepress.com/testimonials.php to read real quotes from published authors. 